\title{
Effects of Lifting Height, Twist Angle and Load mass on Psychophysical Experience, Muscle Activity and Heart Rate during Manual Lifting Isa, H. ${ }^{1,}$, Rawaida, O. ${ }^{1, b}$, Seri, R. K. ${ }^{1, c}$, Muhamad, S. M. ${ }^{1, d}$, Mohd, S. O. ${ }^{1, e}$, Rohana, $\mathrm{A}^{2, \mathrm{f}}$
}

\author{
${ }^{1}$ Department of Manufacturing Management, Faculty of Manufacturing Engineering, Universiti \\ Teknikal Malaysia Melaka, Hang Tuah Jaya, 76100 Durian Tunggal, Melaka, Malaysia. \\ ${ }^{2}$ Faculty of Engineering Technology, Universiti Teknikal Malaysia Melaka Hang Tuah Jaya, 76100 \\ Durian Tunggal, Melaka, Malaysia. \\ aisa@utem.edu.my, bnoorrawaidaomar@gmail.com, 'cseri@utem.edu.my, dsyafiq@utem.edu.my \\ emshahrizan@utem.edu.my, ${ }^{\mathrm{f}}$ rohana_abdullah@utem.edu.my
}

Keywords: Manual lifting, psychophysical experience, muscle activity, heart rate

\begin{abstract}
In industry, manual lifting still a prevalent choice even though mechanized and automated equipment are provided. Mismatch between workers' capability and lifting height, twist angle, and load mass in manual lifting can contribute to occupational injuries such as back pain. The purpose of this study is to investigate the effects of lifting height, twist angle, and load mass on psychophysical experience, muscle activity, and heart rate while performing manual lifting activities. Subjective method associated with Likert Scale was applied to assess the psychophysical experience. Meanwhile, surface Electromyography and heart rate monitor were utilized to measure the muscle activity and heart rate respectively. Main findings of this study show that the rating of psychophysical experience, muscle activity, and heart rate increased when the lifting height and load mass increased. This study concluded that the psychophysical experience and muscle activity were affected when the lifting height, twist angle, and load mass were set at maximum level.
\end{abstract}

\section{Introduction}

Manual lifting is an action of manually grasping an object of definable size and mass with one or two hands, and vertically moving the objects without use of mechanical device. In industries, manual lifting is commonly practiced in material handling activities even though mechanized and automated equipment are facilitated [1]. Improper lifting technique can contribute to occupational injuries such as back pain. Back pain was recorded as a common complaint in Malaysia [2] which can cause absenteeism [3]. Back pain may lead to direct and indirect loses. For example, increase of medical and compensation cost (direct loss), and low productivity due to sickness and absenteeism (indirect loses) [4]. When performing manual lifting, workers may be exposed to bending posture and excessive loads. Overexertion while performing manual lifting was the most frequent occupational injury and illness [5] The Social Security Organization of Malaysia (SOCSO) reported that occupational injuries associated with overexertion due to manual lifting were 545 cases [6]. There are guidelines for safe weight limits to perform manual lifting; however, the guidelines were developed based on European population. Hence the guidelines are not recommended to Malaysian industrial workers due to different in body size and lifting capacity [7]. Furthermore, minimal study been conducted to investigate the effects of load mass, lifting height and twist angle on psychophysical experience, muscle activity and heart rate for Malaysian population. Previous 
studies suggested that physical factors such as load mass, lifting height and twist angle should be critically examined as they can influence the lifting capacity and muscle activity of the workers [8],[9],[10]. The purpose of this study is to investigate the effects of lifting height, twist angle, and load mass on psychophysical experience, muscle activity, and heart rate of female Malaysian.

\section{Methods}

This study has performed 3 tasks: determine the psychophysical experience, analyze the muscle activity, and measure the heart rate of the participants before and after performing manual lifting with various lifting height, twist angle and load mass. Five healthy participants with no history of musculoskeletal problems were selected from the female students at the University Teknikal Malaysia Melaka (UTeM). Two lifting heights $(75 \mathrm{~cm}$ and $140 \mathrm{~cm})$, loads mass $(5 \mathrm{~kg}$ and $10 \mathrm{~kg})$ and twist angles $\left(0^{\circ}\right.$ and $\left.90^{\circ}\right)$ were tested in this study. The lifting frequency was constant at 6 lifts/ min. Participants were given the experimental procedures so that they are clear the steps and protocols in the experimental work. A randomization set of lifting height, load mass and twist angle was performed using Minitab software to reduce opportunities for bias and confounding in experimental design. There were four lifting tests to be performed and each test consists of five replications. Two minutes of time interval for every replication and ten minutes of time interval for each test were given to the participants for the purpose of break and muscle fatigue avoidance [8]. Participants were required to adjust the horizontal lifting distance. They were given sufficient time to get familiar with the experimental procedures before the actual data collection begin. The demographic of the participants were tabulated in Table 1.

Table 1: Demographics of the participants

\begin{tabular}{|l|l|l|l|l|}
\hline Criteria & Min & Mean & Max & Standard Deviation \\
\hline Age $($ year) & 22 & 23.4 & 24 & 0.8 \\
\hline Body mass $(\mathrm{kg})$ & 47 & 60 & 80 & 12.9 \\
\hline Body height $(\mathrm{cm})$ & 156 & 159.2 & 162 & 3.0 \\
\hline
\end{tabular}

\section{Assessment of psychophysical experience}

The psychophysical experience of the participants was determined through subjective assessment associated with Likert Scale (scale of 0 to 4) [11]. Participants were required to fill up personal information such as age, gender, body mass and body height. Participants were required to respond the questions after completing the lifting test by ranking it based on what participants feel during the lifting test. The question is provided with pictures of lower back (erector spinae) and upper arm (biceps).

\section{Analysis of muscle activity}

The surface Electromyography (sEMG) (TeleMyo 2400T G2, Noraxon, USA) and MyoResearch XP Master Edition Software (Noraxon, USA) were used to record, store and analyze all the data obtained through muscle activity measurement. The surface electrode (Ag/AgCl, $10 \mathrm{~mm}$ diameter) was used to measure the muscle activity in the left and right erector spinae and biceps. The muscles were measured throughout the replications. The Non-Invasive Assessment of Muscle (SENIAM) guideline [12] was referred to ensure all the sEMG protocols were complied. The skin of the 
participants was shaved, cleaned and scrubbed with alcohol. The settings of sEMG are as follow: sampling rate: $1,000 \mathrm{~Hz}$; Common mode rejection rate: $110 \mathrm{~dB}$; and Filter: band pass filter (85-500 $\mathrm{Hz}$ ). Throughout the lifting test, participants should immediately report any feeling of pain or difficulties in performing the lifting, so that the sEMG measurement and lifting test could be terminated. The raw signals from the sEMG measurements were rectified in order to make the data positive value using the MyoResearch XP Master Edition software. The rectified signals were analyzed to identify the mean of the signal during lifting test.

\section{Measurement of heart rate}

The heart rate was measured before and after the lifting test by using Heart Rate Monitor (Polar FT2, Finland), measured in beats/min. Participants wore the heart rate monitor on the wrist and the transmitter was attached on the chest. The heart rate of the participants was measured at rest condition (pre-lifting test); and after the lifting test.

\section{Results and Discussion}

\section{Psychophysical Experience}

The following figures illustrate the graph of psychophysical experience of erector spinae (Figure 1) and biceps (Figure 2) versus lifting test. The x-axis shows the lifting test with respect to lifting height, load mass and twist angle. Meanwhile, the y-axis shows the rating of psychophysical experience.

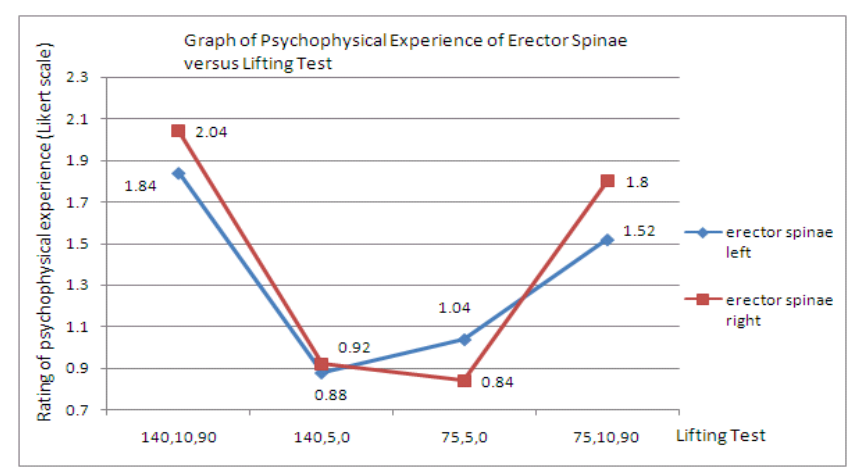

Figure 1: Psychophysical Experience in Erector Spinae versus Lifting Test

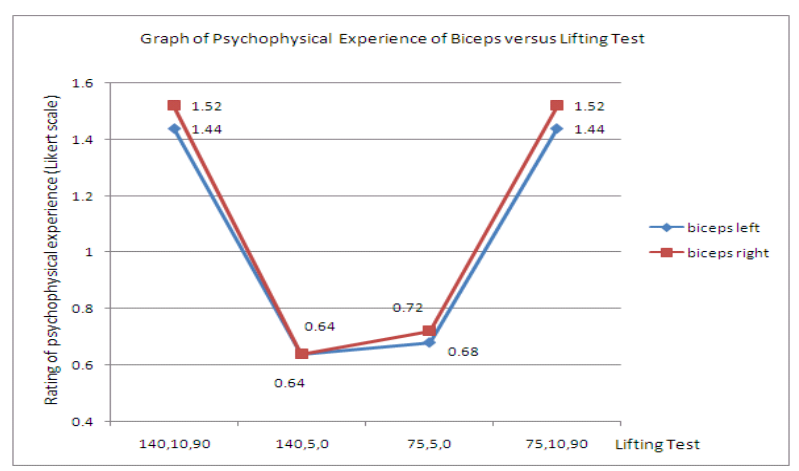

Figure 2: Psychophysical Experience in Biceps versus Lifting Test

Participants experienced moderate pain in the right erector spinae as they need to perform asymmetrical lifting with twist angle $90^{\circ}$ by twisting and bending their body from right with $10 \mathrm{~kg}$ load mass. Figure 2 shows the participants experienced moderate pain in the left and right biceps when performing lifting test at lifting height of $140 \mathrm{~cm}$, twist angle of $90^{\circ}$, and load mass of $10 \mathrm{~kg}$; and when lift load mass of $10 \mathrm{~kg}$, twist angle of $90^{\circ}$ and lifting height of $75 \mathrm{~cm}$. This study pointed that the lifting height, twist angle and load mass affects the psychophysical experience. When the load mass increased, the psychophysical rating increased as well [9]. Wu stated that there is no significant relationship between twist angle and psychophysical experience [8],[10]. However, other studies pointed that performing manual lifting with asymmetric posture (twist angle) is physically difficult and lead to fatigue as the body twist can cause muscle strain [13],[14]. 


\section{Muscle Activity}

The following figures present the muscle activity of biceps (Figure 3) and erector spinae (Figure 4) versus lifting test. The $\mathrm{x}$-axis for both graphs shows the lifting test with respect to lifting height, load mass and twist angle. In the y-axis of Figure 3, it shows the \% of Maximum Voluntary Contraction of biceps, while in the y-axis of Figure 4 indicates the muscle activity (microvolt levels) in the erector spinae.

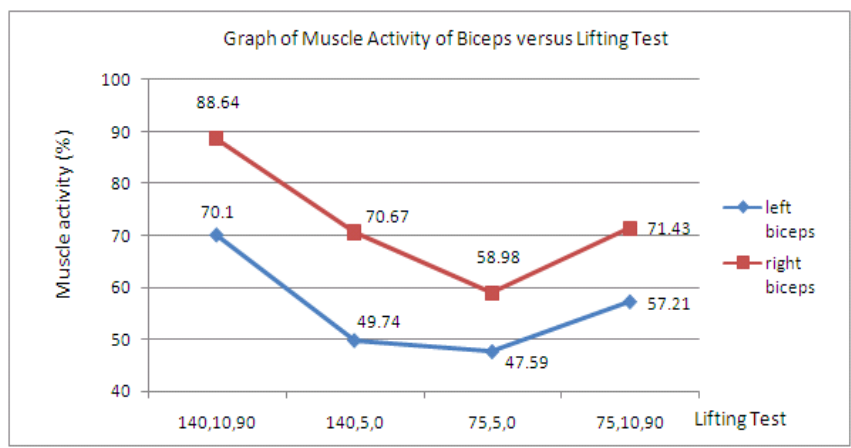

Figure 3: Muscle Activity in Biceps versus Lifting Test

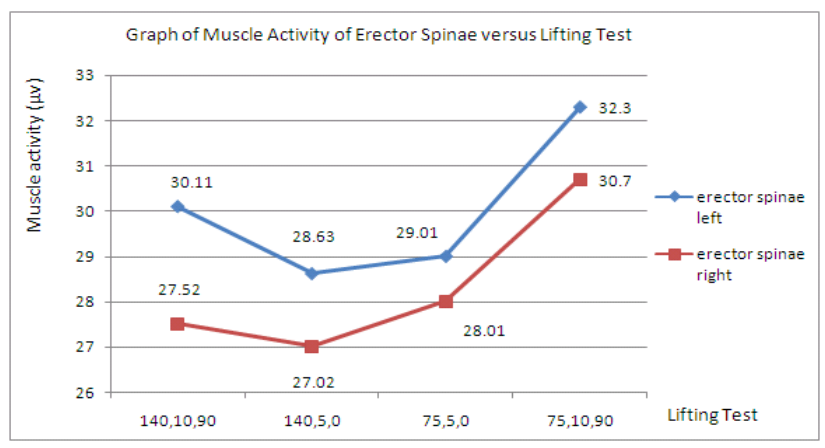

Figure 4: Muscle Activity in Erector spinae versus Lifting Test

Figure 3 and Figure 4 illustrates the trends of muscle activity. It was observed that the muscle activity in the right biceps is higher than the left biceps for all lifting tests. This is due to all participants were right handed. This study found that the left and right biceps experienced highest muscle activity when lifting test was set-up at load mass of $10 \mathrm{~kg}$, twist angle of $90^{\circ}$, and lifting height of $140 \mathrm{~cm}$. Furthermore, the highest microvolt levels were detected in the left and right erector spinae when the participants performed the lifting test at lifting height of $75 \mathrm{~cm}$, load mass of $10 \mathrm{~kg}$, and twist angle of $90^{\circ}$. Meanwhile, the lowest microvolt levels were detected in the left and right biceps as the participants lifted load mass of $5 \mathrm{~kg}$, twist angle of $0^{\circ}$, and lifting height of $75 \mathrm{~cm}$. The findings of this study have a good agreement with previous study whereby the muscle activity in the left and right erector spinae and biceps was significantly affected by the load mass [18]. Additionally, the lifting height also contributed to high muscle activity. This study proved that the muscle activity at biceps was highest when the lifting height is set to maximum level $(140 \mathrm{~cm})$.

\section{Heart Rate}

Figure 5 shows graph of heart rate versus lifting test at before and after performing lifting test. The $\mathrm{x}$-axis indicates the lifting test with respect to lifting height, load mass and twist angle, while the $y$-axis shows the participants' heart rate.

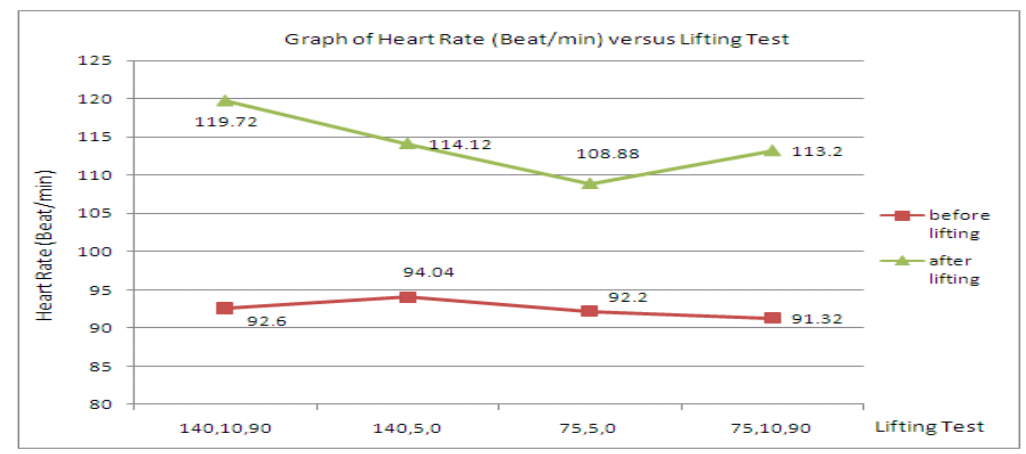

Figure 5: Heart Rate versus Lifting Test 
In all lifting tests, the heart rate of the participants after the test was higher than before the test (Figure 5). The highest heart rate was recorded when the participants are performing the lifting test with load mass of $10 \mathrm{~kg}$, twist angle of $90^{\circ}$, and lifting height of $140 \mathrm{~cm}$. On the other hand, the lowest heart rate was found when the participants are performing the lifting test with load mass of 5 $\mathrm{kg}$, twist angle of $0^{\circ}$, and lifting height of $75 \mathrm{~cm}$. This study proved that the twist angle did not contribute significantly to the heart rate [8],[13]. In contrast, the load mass and lifting height have a significant influence in heart rate [15].

\section{Conclusion}

This study has conducted a laboratory based experiment to investigate the effects of lifting height, twist angle, and load mass on psychophysical experience, muscle activity, and heart rate of female Malaysian. The psychophysical experience and muscle activity were affected when the lifting height, twist angle, and load mass were set at $140 \mathrm{~cm}, 90^{\circ}$ and $10 \mathrm{~kg}$ respectively. Additionally, the heart rate was influenced by lifting height of $140 \mathrm{~cm}$ and load mass of $10 \mathrm{~kg}$. Based on these findings, this study concluded that the psychophysical experience and muscle activity were most affected by lifting height, twist angle and load mass. Meanwhile, heart rate only affected by the lifting height and load mass. In the future research works, this study suggests to consider the effects of environmental factors such as temperature and humidity on human psychology and physiology during manual lifting.

\section{Acknowledgment}

The authors would like to acknowledge the Universiti Teknikal Malaysia Melaka (UTeM) for funding this research under University Short Term Grant (PJP/2012/FKP(12C)/S01016 and the Faculty of Manufacturing Engineering (UTeM) for providing facilities in carrying out this study.

\section{References}

[1] C.C. Chang, S. Hsiang, P.G. Dempsey, and R.W. Mcgrorry, A computerized video coding system for biomechanical analysis of lifting task, Int. J. of Industrial Ergonomics 32 (2003) 239-250.

[2] K. Veerapen, R.D. Wigley and H. Valkenburg, Musculoskeletal pain in Malaysia: A COPCORD Survey, The Journal of Rheumatology (2007) 207-213.

[3] B. Widanarko, S. Legg, M. Stevenson, J. Devereux, A. Eng, A. Mannetje, S. Cheng, N. Pearce, Applied Ergonomics 43 (2012) 727-737.

[4] FG. Benavides, Ill Health, Social protection, labour relations, and sickness absence, Occup Environ Med 63 (2006) 221-229.

[5] Bureau of Labor Statistics (2010), information on www.bls.gov.

[6] Social Security Organization of Malaysia (SOCSO) Annual Report, 2010.

[7] S.P. wu, Maximum acceptable weight for asymmetric lifting of Chinese females, Applied Ergonomics 34 (2003) 215-224.

[8] S.M. Alavinia, DucoMolenaar and A. Burdorf, Productivity loss in the workforce: Association with Health, Work Demands, and Individual Characteristics, American J. of Ind. Med. (2008) 1-8 
[9] A.S. Jackson, G. Borg, J.J. Zhang, K.R. Luaghery, and J. Chen, Role of physical work capacity and load mass on psychophysical lift ratings, Int. J. of Ind. Ergonomics 20 (1997) 181-190.

[10] S.P. Wu, Psychophysical determined symmetric and asymmetric lifting capacity of Chinese males for one hour's work shift, Int. J. of Ind. Ergonomics 25 (2000) 675-682.

[11] I. Halim, A.R. Omar, A.M. Saman and I. Othman, Assessment of fatigue associated with prolonged standing in the workplace, Safety Health Work 3 (2012), 31-42.

[12] B. Freriks and H. Hermens, European recommendation for surface electromyography, result of SENIAM Project," Roessignh Research and Development, The Netherlands (2000).

[13] A. Mital, and H.F. Fard, Psychophysical and physiological response to lifting symmetrical and asymmetrical loads, Ergonomics 29 (1986) 1263-1272.

[14] J. Yoon, A. Shiekhzadeh and M. Nordin, The effect of load weight vs. pace on muscle recruitment during lifting, Applied Ergonomics 43 (2012) 1044-1050.

[15] N. Singh, R.M. Belokar and R.S. Walia, Physiological evaluation of manual lifting task on Indian male workers, Int. J. of Eng. and Adv. Tech.Vol.2 Iss. (2012) 8-16. 